\title{
ON THE LUMINOSITY FUNCTION OF ELLIPTICAL GALAXIES
}

\author{
V.S. Popov \\ Central Astronomical Observatory \\ of the USSR Academy of Sciences \\ 196140 Leningrad, Pulkovo \\ USSR
}

Originally the luminosity function of galaxies was derived by E. Hubble (1936). Later studies are by Holmberg (1950), Zwicky (1957, 1964), Kiang (1961), Neyman and Scott (1962), Van den Bergh (1961), Pskovsky (1965), Nezhinsky and Osipkov (1967, 1969), Genkina (1969), Popov (1980), Tammann (1984), and others.

Luminosities of elliptical galaxies are within the range $-10^{m} .0$ to $-24^{m} .0$ with a maximum at $M_{v}=-19^{m} .0$. They are very close to the integral luminosity function of globular clusters of the Galaxy with the mode $M_{v}=-7^{m} .0$, i.e. they converge with weak dwarf galaxies of the Local group of galaxies.

If these observational data are combined with the luminosity function of stars in the Galaxy, with the mode $M_{v}=+14^{m} .0$, a new observational fact can be derived.

\section{TABLE}

\begin{tabular}{lcccc}
\hline \hline Object & $\mathcal{M}\left(M_{\odot}\right)$ & $3 \Delta \log \mathcal{M}$ & Mode $M_{v}$ & $\Delta M_{v}$ \\
\hline Stars & 1 & & 14 & 21 \\
Globular Clusters & $10^{7}$ & 21 & -7 & 12 \\
Elliptical Galaxies & $10^{11}$ & 12 & -19 & \\
\hline
\end{tabular}

Each group of objects (stars, globular clusters, elliptical galaxies) has its own maximum on the common curve of the luminosity function. If these maxima are compared with the characteristic mass of the matter in these objects, then we discover $\Delta M_{v}=3 \Delta \log \mathcal{M}$, where $M$ is the absolute magnitude of the object, and $\mathcal{M}$ is its characteristic mass. 


\section{REFERENCES}

Hubble, E.P., 1936. The Realm of the Nebulae, New Haven.

Holmberg, E., 1950. Lund Medd., No. 128.

Zwicky, F., 1957. Morphological Astronomy, Berlin.

Zwicky, F., 1964. Astroph. J., 140, 1626.

Kiang, T., 1961. M.N.R.A.S., $122,262$.

Neyman, J., \& Scott, E., 1962. A.J., 67, 119, 582.

van den Bergh, S., 1961. Z. für Astroph., 53, 219.

Pskovsky, Yu.P., 1965. Vestnik MGU, 1.

Nezhinsky, E.M., \& Osipkov, L.P., 1967. Trudy AO LGU 24, 117.

Nezhinsky, E.M., \& Osipkov, L.P., 1969. Trudy AO LGU 26, 92.

Genkina, L.M., 1969. Trudy Astroph. Inst. AN Kaz., SSR, 12, 106.

Popov, V.S., 1980. Izvestia GAO AN SSSR, 198, 15.

Tammann, G.A., 1984. The Hubble Diagram, in Clusters and Groups of Galaxies, 529 (Dordrecht). 